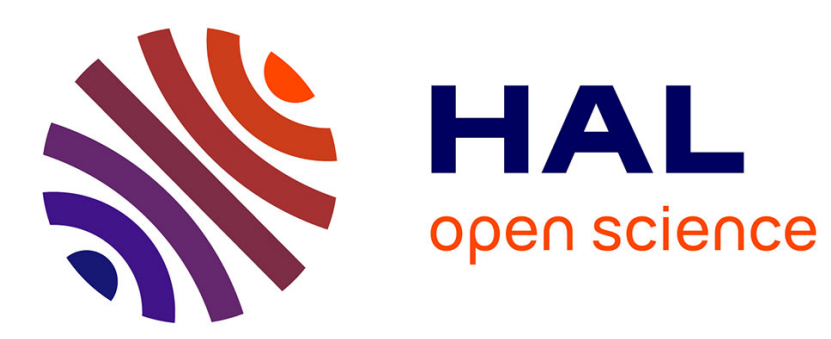

\title{
On Apparent Irrational Behaviors: Interacting Structures and the Mind
}

Pierre Gosselin, Aïleen Lotz, Marc Wambst

\section{To cite this version:}

Pierre Gosselin, Aïleen Lotz, Marc Wambst. On Apparent Irrational Behaviors : Interacting Structures and the Mind. 2013. hal-00851309

\section{HAL Id: hal-00851309 \\ https://hal.science/hal-00851309}

Preprint submitted on 13 Aug 2013

HAL is a multi-disciplinary open access archive for the deposit and dissemination of scientific research documents, whether they are published or not. The documents may come from teaching and research institutions in France or abroad, or from public or private research centers.
L'archive ouverte pluridisciplinaire HAL, est destinée au dépôt et à la diffusion de documents scientifiques de niveau recherche, publiés ou non, émanant des établissements d'enseignement et de recherche français ou étrangers, des laboratoires publics ou privés. 


\title{
On Apparent Irrational Behaviors : Interacting Structures and the Mind
}

\author{
P. Gosselin ${ }^{1}$, A. Lotz ${ }^{2}$, and M. Wambst ${ }^{3}$ \\ ${ }^{1}$ Institut Fourier, UMR 5582 CNRS, Université de Grenoble I, France \\ ${ }^{2}$ Cerca Trova, Grenoble, France \\ ${ }^{3}$ IRMA, UMR 7501 CNRS, Université de Strasbourg, France
}

June 29, 2013

\begin{abstract}
We develop a general method to solve models of interactions between multiple and possibly strategic agents. Our model explains apparently irrational or biased behaviors in a person. We argue that these actions could result from several rational structures having different goals. Our main example is a model of three agents, "conscious", "unconscious", and "body". Our main result states that, for an agent whose unconscious and conscious goals differ, the unconscious may influence the conscious, either directly or indirectly, via a third agent, the body. This three-agent model describes behaviors such as craving, excessive smoking, or sleepiness, to delay or dismiss a task. One of the main result shows that the unconscious' strategic action crucially depends on whether the conscious' actions are complementary in time. When complementary, and if the conscious is not sensitive to unconscious' messages, the unconscious may drive the conscious towards its goals by blurring physical needs. When not complementary, the unconscious may more easily reach his goal by influencing the conscious, be it directly or indirectly.
\end{abstract}

Keywords: dual agent; conscious and unconscious; rationality; multi-rationality; consistency; choices and preferences; multi-agent model.

JEL Classification: C02, C65, C70, D01, D87.

\section{Introduction}

When economics first became identified as a distinct field of study, psychology did not exist as a discipline. When it finally did, economists distanced themselves from it: psychology was seen as not sufficiently founded to provide the laws economics needed to impose itself as a natural science. The discovery of the unconscious, at the turn of the $\mathrm{XX}^{\text {th }}$ century, further contributed to this entrenchment.

It was only in the face of the repeated failures of their predictions, that economists were poised to depart from modeling the "perfect rational man". Today, the irrationality displayed by agents is seen as the main culprit of the repeated failures of economic models. By taking into account the "true" behavior of agents, i.e. by acknowledging the fact that they do not systematically act as fully rational agents, it is hoped that the flaws of economic predictions will be corrected. This approach is at the root of behavioral economics. Combining psychology 
and economics, it investigates what happens in markets in which some of the agents display human limitations and complications [MT01].

By providing economics with more realistic psychological foundations, behavioral economics attempts to increase the explanatory power of economics. Anomalies in standard economic normative assumptions are identified, used to create alternative theories that generalize existing models and, more recently, directly included in economic models of behavior to derive fresh implications, and test them. The modification of one or two assumptions in standard theory in the direction of greater psychological realism suffice. It is stated that, often, these departures are not radical at all because they relax simplifying assumptions that are not central to the economic approach [CL03].

Yet this approach has a flaw: although pinpointing anomalies is certainly valid to underline the failures of a model, and using them as stylized facts to modify rational assumptions may improve the predictive power of economic models, this approach is unlikely to improve our understanding of our bounded rationality and irrational behaviors.

Most importantly, the behavioral approach does not question the nature of the information process at the unconscious level. In other words, it fails to question under which conditions the unconscious could be modeled as fully and plainly rational, and the consequences this assumption could have. This approach to the unconscious processes is therefore partial. It represents a shortcut to the true question: to understand human behavior, one has to suppose it is indeed rational in the plain economic sense of the term.

Merely acknowledging our psyche as rational but somehow "bounded" dismisses the possibility of a specific and fully rational agent, the unconscious, continuously acting alongside the conscious within ourselves. Needless to say that this reasoning is understandable. One could argue that, if the unconscious was indeed a rational agent, having the same information as the conscious, the behavior of the individual as a whole, i.e. the sum of two rational and identically informed agents, should be unique and rational. Since it is not, one concludes that the unconscious is not fully rational. Hence the departure from full rationality.

The two first authors have argued elsewhere [Lot11, GL12] that, as long as some unconscious activity takes place within our minds, it must be modeled as a fully rational agent, in the economic sense of the term. They furthermore inferred that it should act permanently, i.e. that it cannot be "dismissed" at will. Finally, its presence and its action must be considered as fully rational, and in consequence rely on an alternate set of information.

Crucially, irrationality in unconscious-led behavior, in our view, does not arise from the fact that the unconscious is irrational, but rather from the fact that these two agents evaluate events through different grids, and act through one single body. This approach has been developed in [Lot11], who dubbed the resulting agent, i.e. the combination of two fully economically rational agents, the "dual agent". Building on the dual-agent approach, [GL12] showed that, under specific conditions, the dynamics of the conscious and unconscious seen as two autonomous and interacting structures may display instability, depending on the structures interactions strength. However, if unconscious has a strategic advantage, greater stability is reached. This result shows that some switch in the conscious' objective can appear in our setting. Behaviors that can't be explained with a single utility can thus turn out to be rational if a rational unconscious agent is added. Our results justify our hypothesis of a rational interacting unconscious. It supports the widening of the notion of rationality to multi-rationality in interaction.

True, the strategy of breaking up the person into interacting sub-agents is being explored to economically model inconsistent people [Ros10]. Dual or multiple selves acting "synchronically" and/or "diachronically" on a similar set of information and having different objectives have already been shown to provide a unified explanation for several behavioral anomalies ${ }^{1}$, among

\footnotetext{
${ }^{1}$ See [Str56, Sch78, Sch80, Sch83, Ain92, Ain01, Ros05, Ros09]
} 
which cases of procrastination such as Akerlof's [Ake91]. Ainslie's picoeconomic framework represents this situation as a bargaining game between several selves [Ain92] (see [Ros10] and references therein for an account).

The notion of dual agent must specifically be distinguished from the dual self considered in Fudenberg and Levin [FL06]. The latter consists of sub-structures, both long run and short run, to model behaviors of self control. In these authors' perspective, the long run self imposes costs on short run selves and controls their behaviors. Their approach differs from ours on two points. First, they model a successive identification of the agent to several selves, each one disappearing in turn. We believe, on the contrary, that a pattern of behavior displays a certain persistency and must, as a consequence, be active at all stage of the decision making.

We argue that the human psyche can be modeled as several agents in complex, simultaneous, and possibly strategic, interactions. Importantly, in [FL06] and in picoeconomics in general, the role of the unconscious - inasmuch such a notion can be attributed to the sub-structures they consider - appears as a mere unknown random modification on the short term utility. Unknown mechanisms induce a change in the agent utility. These mechanisms are precisely the ones we want to describe, along with what makes these actions inconsistent or incoherent. Only by describing some unconscious rational behaviors, can we hope to deal with these problems.

A step in this direction was made in [GL12] where, following [Lot11], two types - dominating strategic/non strategic - of agents were considered. In particular, [GL12] presented an example of a two-agent model of direct interaction between a person's conscious and unconscious. However the authors acknowledged that several interacting strategic agents may be at the root of the decision process. This paper therefore presents a mathematical framework including several strategic agents linked by complex hierarchical interconnections. It models interactions where agents can have a strategic advantage over some agents, while being dominated by others. In particular, the framework includes the possibility of several competing agents with contradictory objectives. As an example, we study a model with three agents, "conscious", "unconscious" and "body" respectively, where the unconscious triggers body needs to indirectly alter the person's conscious goals. We suggest that, under certain conditions, such a model can well describe several behaviors, such as hyperactivity or depression, etc ...

The paper is organized as follows. The first section presents a one-period model of three interacting agents. Several decision centers, i.e., economic agents, are encapsulated in one agent, and each center has specific autonomy, and potentially conflicting goals and strategies. A characteristic feature of the model is the strategic advantage some agents have over others, that provides the description of a person's behavior when his/her conscious will, unconscious drive and body's needs conflict.

The second section solves the model, presents the equilibrium, interprets the solutions and describe the relevant behaviors that can be described by our formalism. The third section extends the model to an arbitrary number of interacting agents. Here again some agents have strategical advantage over some others. An explicit recursive resolution process is provided for this general setting. As an example we recover the solutions of the three interacting agents model. The last section concludes. Possible applications of the general model are briefly considered.

\section{The three agents model}

This section presents a static model of three interacting agents. Its aim is to describe apparently irrational and incoherent behaviors, seen as the resulting action of three relatively independent 
sub-structures, i.e agents, among the same psyche. This model will serve as an introductory example of the general formalism of interacting agents of Section 3.

Practically, we model an agent whose conscious wants to perform a task. However, his unconscious has other goals and may consider this specific task as non optimal. Moreover, a third agent, denoted "the body", has specific needs that have to be satisfied. We assume that the unconscious manipulates both the body and the conscious by sending signals, such as discomforts or strains. This models builds on [LG], but here the novelty consists in inspecting the indirect action of the unconscious on the conscious, via the body and its needs.

Let us consider the hypothetical case, where a student, involved in the precise task of learning, experiences difficulties in concentrating. Each time he starts working, he is quickly overwhelmed by sleepiness, boredom or compulsions, such as smoking or eating. We will consider that these compulsions appear at these specific moments, when the student is both rested and fed, and as such, cannot correspond to objective physical needs. We see in this "coincidence" a warning message that signals the action of the unconscious. In our perspective, the unconscious has other priorities and goals, and moreover his interpretation grid differs from the conscious' one (see [GL12] for more details). The conscious actions are read by the unconscious. Interpreted according to a completely different grid, they are understood as suboptimal or potentially dangerous by the unconscious. In the case of our student, we can suppose that, for some reasons, the unconscious perceives learning as harmful, and will use its power to thwart the conscious action.

The student's visible behavior is merely the result of the three agents' interactions. In a dynamical perspective, it may lead to some incoherent patterns, such as inconsistency in an agent's goal .

\subsection{The agent's utility functions}

Let us now describe more precisely the model.

Throughout the paper, C, B, and $\mathrm{U}$ will respectively stand for the conscious, body and unconscious agents. We model the body as an automaton whose action is a signal $n$ of a physical need. By convention, a positive $n$ will describe a need, while negative $n$ will stand for a satiety signal. The agent $\mathrm{C}$, the conscious, has two possible actions. It performs a task $t$, which optimum is arbitrarily normalized to 0 . But he can also respond to agent B's signal with a second action $f$, for feeding.

The model considers two possibilities. In the first case, the two actions are non exclusive, and no constraint is imposed on them. In the second case, a time constraint do exist, and both action are exclusive and complementary. The agent's time is divided optimally between the two actions.

For the unconscious, agent $\mathrm{U}$, the optimum is not $t=0$, but an other value $\tilde{t}$. In order to manipulate $\mathrm{C}$ and have its optimum changed, $\mathrm{U}$ can send three strains. Two of them, $s_{t}$ and $s_{f}$, directly affect $\mathrm{C}$ in reaction to its actions $t$ and $f$. The last one, $s_{n}$ influences $\mathrm{B}$ in reaction to $n$.

Finally, we suppose that, although they are influenced by the unconscious strains, $\mathrm{C}$ and $a$ fortiori B are not aware of the presence, and goals, of U.

To model the situation at stake, the three agents $\mathrm{B}, \mathrm{U}$ and $\mathrm{C}$ utilities will be quadratic and denoted $U_{\mathrm{B}}, U_{\mathrm{U}}, U_{\mathrm{C}}$ respectively, such that: 


$$
\begin{aligned}
U_{\mathrm{B}} & =-\frac{1}{2}(n+f)^{2}-\alpha n s_{n} \\
U_{\mathrm{U}} & =-\frac{1}{2}\left(\beta(f-\tilde{f})^{2}+\gamma(t-\tilde{t})^{2}+s_{n}^{2}+s_{f}^{2}+s_{t}^{2}\right) \\
U_{\mathrm{C}} & =-\frac{1}{2}\left(t^{2}+\delta n^{2}\right)-\nu n t-\kappa s_{f}(f-\tilde{f})-\eta s_{t}(t-\tilde{t})
\end{aligned}
$$

As usual in this kind of model, these utilities will be quadratic, and normalized so that the quadratic terms coefficients in the control variables are $-\frac{1}{2}$ or 0 (in the utility of each agent).

The body is merely considered as an automaton. It has no specific goals, and its utility function $U_{\mathrm{B}}$ describes its reaction to other agents' actions. In this setting, endowing the body with specific goals would have allowed it to manipulate the conscious, which was not our purpose here. Without any interaction with the unconscious $\mathrm{U}$, the body would react, in first approximation, linearly to the feeding provided by the conscious $\mathrm{C}$. This led us to state the first term $-\frac{1}{2}(n+f)^{2}$ in $U_{\mathrm{B}}$. The influence of the unconscious on the body is described as a perturbation $-\alpha n s_{n}$, sent by the unconscious as a reaction to the physical signal. Without this term, one can easily check that B's optimum is reached for $n=-f=0$. This result being suboptimal for $\mathrm{U}$, he will react to drive the equilibrium toward his own goal $\tilde{f}$.

The response $n$ of $\mathrm{B}$ does not depend directly on the task $t$ performed by $\mathrm{C}$, in line with our previous hypothesis that this task is not physically demanding. We are not interested in modeling the physical strain, but will rather study the use of the body by the unconscious to reach his goal, i.e. how $\mathrm{U}$ can break an equilibrium between $\mathrm{B}$ and $\mathrm{C}$. By convention, we will impose the parameter $\alpha$ to be positive, so that a positive strain will respond to a positive feeding.

Now, consider $U_{\mathrm{C}}$, agent C's utility. Its first term $-\frac{1}{2} t^{2}$ encompasses C's utility in the absence of both the unconscious $\mathrm{U}$ and the body B. In this - hypothetic - situation, without any constraint on $t$, agent $\mathrm{C}$ would optimally choose $t=0$.

The terms $-\frac{1}{2} \delta n^{2}-\nu n t$ describe the effect of the hunger signal on C. The higher the need, the more painful is the task. Let us anticipate here by mentioning that in the absence of $\mathrm{U}$, agent $\mathrm{C}$ will still be able to set $t=0$ by adjusting the feeding to the anticipated need.

The term $-\frac{1}{2} \delta n^{2}$ models the fact that, even without any work being involved, the need is, in itself, painful. As a consequence, we will acknowledge this fact by choosing $\delta>0$. This a direct consequence of dismissing any cost to the feeding $f$. Usually, models would impose costs to an agent's tasks, or some constraints between these tasks .

In other word, without $\mathrm{U}$, the body $\mathrm{B}$ and the variable $f$ could be discarded from C's equilibrium. When included, $\mathrm{U}$ indirectly manipulates $\mathrm{C}$ through $\mathrm{B}$ by assigning a strategic role to $f$. We will however consider the inclusion of a binding constraint on the feeding, by considering two alternative models. In the first one, we will consider $f$ and $t$ as complementary activities within a given time span, and will set a binding constraint such that $f+t=1$. In the alternative model, the variables $f$ and $t$ will be considered independent.

The terms $-\kappa s_{f}(f-\tilde{f})-\eta s_{t}(t-\tilde{t})$ are the perturbations induced by $\mathrm{U}$, in its attempt to impose his goals $\tilde{f}$ and $\tilde{t}$ on C. These terms actually drive C's actions from 0 toward $\tilde{f}$ and $\tilde{t}$.

Some additional technical conditions on $U_{\mathrm{C}}$ will be convenient. Setting $\delta-\nu^{2}>0$ will ensure that $U_{\mathrm{C}}$ is negative definite, and allow the existence of an optimum. Furthermore, imposing 
$\nu>0$ will imply that for $n>0$ and $t>0$, agent $\mathrm{C}$ suffers a loss in its utility, as one can expect from a combination of both a need non satisfied associated with excessive working.

Agent U's utility $U_{\mathrm{U}}$ displays two terms $-\frac{1}{2} \beta(f-\tilde{f})^{2}-\frac{1}{2} \gamma(t-\tilde{t})^{2}$ that clearly model U's goal with respect to C's activity. U considers certain levels of the conscious' activity, $\tilde{f}, \tilde{t}$ as optimal. Let us stress, as explained in [LG], that an agent facing a situation, whether the conscious or unconscious, builds his interpretation, and thus his utility function, through his own grid of lecture. As a consequence, $\mathrm{U}$ and $\mathrm{C}$ have a complete different interpretation of a current situation. We will impose $\beta$ and $\gamma$ to be positive, so that $U_{\mathrm{U}}$ can have an optimum.

The remaining terms $-\frac{1}{2}\left(s_{n}^{2}+s_{f}^{2}+s_{t}^{2}\right)$ model the cost incurred by the strains imposed on other agents. Since we did postulate that the three agents are one single individual, a strain inflicted by one agent is indeed painful for all of them.

Some additional constraints on the coefficients and the variables are required to have a realistic model. Because agent $\mathrm{U}$ sends a pain if his goals are not reached, $s_{f}$ et $s_{t}$ are assumed positive. We do not impose such a constraint on $s_{n}$, since no optimum value for $n$ is expected by U. Being an adjustment variable, $s_{n}$ can be seen as a pain as well as a reward for the body.

To model this pain, the terms $-\kappa s_{f}(f-\tilde{f})$ and $-\eta s_{t}(t-\tilde{t})$ should be negative. We therefore impose $\tilde{f}$ and $\kappa$ (respectively $\tilde{t}$ and $\eta$ ) to have opposite signs. This comes directly from the fact that the equilibrium values of $f$ and $t$ will obviously lie between 0 and $\tilde{f}$ and respectively between 0 and $\tilde{t}$.

We will furthermore assume that some agents have a strategical advantage over others. Recall first that B, the body, has been considered as an automaton, and as such is not a strategic agent. Given the psychological situation we had in mind (neurosis, phobia, etc) we assume that the unconscious $\mathrm{U}$ has a strategic advantage over the conscious $\mathrm{C}$. Both $\mathrm{U}$ and $\mathrm{C}$ are strategic with respect to B, and can thus manipulate him. This situation can be modeled by an oriented graph whose vertices are $\mathrm{B}, \mathrm{C}$, and $\mathrm{U}$. The orientation of the edges of the graphs summarize the strategic advantages: $\mathrm{C} \rightarrow \mathrm{B}, \mathrm{U} \rightarrow \mathrm{B}, \mathrm{U} \rightarrow \mathrm{C}$. Later a formalization with help of graphs will allow us to describe and solve models for an arbitrary number of interacting agents.

As usual the hierarchy of strategical advantages will determine the order of resolution. This will be detailed in the next section. As quoted before, we will consider two cases. We first implement the time binding constraint $t+f=1$ for the conscious agent actions. In a second time, we suppose that there is no such constraint and that $n$ and $t$ are independent.

\subsection{The case $f+t=1$}

We replace directly $f=1-t$ in the utility functions:

$$
\begin{gathered}
U_{\mathrm{B}}=-\frac{1}{2}(n+1-t)^{2}-\alpha n s_{n} \\
U_{\mathrm{U}}=-\frac{1}{2} \beta(1-t-\tilde{f})^{2}-\frac{1}{2} \gamma(t-\tilde{t})^{2}-\frac{1}{2} s_{n}^{2}-\frac{1}{2} s_{n}^{2}-\frac{1}{2} s_{t}^{2} \\
U_{\mathrm{C}}=-\frac{1}{2} t^{2}-\frac{1}{2} \delta n^{2}-\nu n t-\kappa s_{f}(1-t-\tilde{f})-\eta s_{t}(t-\tilde{t})
\end{gathered}
$$

We will adopt a matricial formalism for the utility functions. Up to a constant number, the 
utilities can be written as:

$$
\begin{aligned}
U_{\mathrm{B}} & =-\frac{1}{2}{ }^{t} X_{1} X_{1}-{ }^{t} X_{1} A_{12}^{(1)} X_{2}-{ }^{t} X_{1} A_{13}^{(1)} X_{3}-\frac{1}{2}{ }^{t} X_{3} A_{33}^{(1)} X_{3}+\left({ }^{t} X_{3}-{ }^{t} X_{1}\right) E_{1} \\
U_{\mathrm{U}} & =-\frac{1}{2}{ }^{t} X_{2} A_{22}^{(2)} X_{2}-\frac{1}{2}{ }^{t}\left(X_{3}-\tilde{X}_{3}\right) A_{33}^{(2)}\left(X_{3}-\tilde{X}_{3}\right) \\
U_{\mathrm{C}} & =-\frac{1}{2}{ }^{t} X_{3} A_{33}^{(3)} X_{3}-\frac{1}{2}{ }^{t} X_{1} A_{11}^{(3)} X_{1}-{ }^{t} X_{3} A_{31}^{(3)} X_{1}-{ }^{t} X_{2} A_{23}^{(3)} X_{3}-{ }^{t} X_{2} \check{A}_{23}^{(3)} \check{X}_{3}
\end{aligned}
$$

where the control variables for the agents are concatenated in the following vectors:

$$
X_{1}=(n), \quad X_{2}=\left(\begin{array}{c}
s_{n} \\
s_{t} \\
s_{f}
\end{array}\right), \quad X_{3}=(t) .
$$

The left upper-script ${ }^{t}(\cdot)$ denotes the usual transposition of matrices. The goals of $\mathrm{U}$ are enclosed in $\tilde{X}_{3}=\left(\frac{\gamma \tilde{t}+\beta(1-\tilde{n})}{\beta+\gamma}\right)$. Recall that all others goals are normalized to 0 . We also set $\check{X}_{3}=\left(\begin{array}{c}-\tilde{t} \\ 1-\tilde{f}\end{array}\right)$. The utilities quadratic relations are commanded by the following parameters matrices.

$$
\begin{gathered}
A_{12}^{(1)}=\left(\begin{array}{ccc}
\alpha & 0 & 0
\end{array}\right), \quad A_{13}^{(1)}=(-1), \quad A_{33}^{(2)}=(\beta+\gamma) \\
A_{33}^{(3)}=(1), \quad A_{11}^{(3)}=(\delta), \quad A_{31}^{(3)}=(\nu) \\
A_{33}^{(1)}=(1), \quad E_{1}=(1), \\
A_{22}^{(2)}=\left(\begin{array}{lll}
1 & 0 & 0 \\
0 & 1 & 0 \\
0 & 0 & 1
\end{array}\right), \quad A_{23}^{(3)}=\left(\begin{array}{c}
0 \\
\eta \\
-\kappa
\end{array}\right), \quad \check{A}_{23}^{(3)}=\left(\begin{array}{ll}
0 & 0 \\
\eta & 0 \\
0 & \kappa
\end{array}\right)
\end{gathered}
$$

By convention, we set $A_{j i}^{(i)}={ }^{t}\left(A_{i j}^{(i)}\right)$.

Since $\mathrm{B}$ is dominated by both $\mathrm{C}$ and $\mathrm{U}$, and $\mathrm{C}$ is dominated by $\mathrm{U}$, the following matricial formulas will lead to the same equilibrium.

$$
\begin{aligned}
U_{B}^{\prime} & =-\frac{1}{2} X_{1} X_{1}-{ }^{t} X_{1} A_{12}^{(1)} X_{2}-{ }^{t} X_{1} A_{13}^{(1)} X_{3}-\frac{1}{2}{ }^{t} X_{3} A_{33}^{(1)} X_{3}-{ }^{t} X_{1} E_{1} \\
U_{U}^{\prime} & =-\frac{1}{2} X_{2} A_{22}^{(2)} X_{2}-\frac{1}{2}{ }^{t}\left(X_{3}-\tilde{X}_{3}\right) A_{33}^{(2)}\left(X_{3}-\tilde{X}_{3}\right) \\
U_{C}^{\prime} & =-\frac{1}{2} X_{3} A_{33}^{(3)} X_{3}-\frac{1}{2}{ }^{t} X_{1} A_{11}^{(3)} X_{1}-{ }^{t} X_{3} A_{31}^{(3)} X_{1}-{ }^{t} X_{2} A_{23}^{(3)} X_{3}
\end{aligned}
$$

\subsubsection{Resolution}

Let us now solve the optimization problem. Agent B having no strategical advantage, we will solve his optimization problem first. One obtains directly 


$$
X_{1}=-\left(A_{12}^{(1)} X_{2}+A_{13}^{(1)} X_{3}+E_{1}\right)
$$

which expresses $X_{1}$ as a function of $X_{2}$ and $X_{3}$. Replacing this expression in $U_{\mathrm{C}}^{\prime}$ and $U_{\mathrm{U}}^{\prime}$, we follow the hierarchy of strategical advantages, and optimize $U_{\mathrm{C}}^{\prime}$ on $X_{3}$. It yields

$$
A_{33}^{(3)} X_{3}+A_{32}^{(3)} X_{2}-A_{31}^{(1)} A_{11}^{(3)} X_{1}+A_{31}^{(3)} X_{1}-{ }^{t} X_{3} A_{31}^{(3)} A_{13}^{(1)}=0
$$

from which $X_{3}$ is obtained:

$$
X_{3}=K X_{2}+E_{3}
$$

with

$$
\begin{aligned}
K & =-\left(A_{33}^{(3)}+A_{31}^{(1)} A_{11}^{(3)} A_{13}^{(1)}-A_{31}^{(3)} A_{13}^{(1)}-A_{31}^{(1)} A_{13}^{(3)}\right)^{-1}\left(A_{32}^{(3)}+A_{31}^{(1)} A_{11}^{(3)} A_{12}^{(1)}-A_{31}^{(3)} A_{12}^{(1)}\right) \\
E_{3} & =-\left(A_{33}^{(3)}+A_{31}^{(1)} A_{11}^{(3)} A_{13}^{(1)}-A_{31}^{(3)} A_{13}^{(1)}-A_{31}^{(1)} A_{13}^{(3)}\right)^{-1}\left(A_{31}^{(1)} A_{11}^{(3)}-A_{31}^{(3)}\right) E_{1} .
\end{aligned}
$$

Alongside with $X_{3}$, we rewrite $X_{1}$ as a function of the sole variable $X_{2}$ and get

$$
\begin{aligned}
X_{1} & =-\left(A_{12}^{(1)} X_{2}+A_{13}^{(1)} X_{3}\right)-E_{1} \\
& =L X_{2}-A_{13}^{(1)} E_{3}-E_{1}
\end{aligned}
$$

where

$$
L=-\left(A_{12}^{(1)}+A_{13}^{(1)} K\right) .
$$

Ultimately, the optimization of $U_{\mathrm{U}}^{\prime}$ leads to a complete resolution of the model in function of the parameters and the goals $\tilde{X}_{3}$ in the following way:

$$
\begin{aligned}
& X_{2}=\left(A_{22}^{(2)}+{ }^{t} K A_{33}^{(2)} K\right)^{-1}{ }^{t} K A_{33}^{(2)} \tilde{X}_{3}+E_{2} \\
& X_{3}=K\left(A_{22}^{(2)}+{ }^{t} K A_{33}^{(2)} K\right)^{-1}{ }^{t} K A_{33}^{(2)} \tilde{X}_{3}+K E_{2}+E_{3} \\
& X_{1}=L\left(A_{22}^{(2)}+{ }^{t} K A_{33}^{(2)} K\right)^{-1}{ }^{t} K A_{33}^{(2)} \tilde{X}_{3}+L E_{2}-A_{13}^{(1)} E_{3}-E_{1}
\end{aligned}
$$

with $E_{2}=-\left(A_{22}^{(2)}+{ }^{t} K A_{33}^{(2)} K\right)^{-1}{ }^{t} K A_{33}^{(2)} E_{3}$.

Setting

$$
V=\nu+1, \quad W=\nu+\delta, \quad \Gamma=\gamma+\beta, \quad \text { and } \quad R=\frac{\kappa^{2}+\eta^{2}}{\alpha^{2}}
$$

we obtain 


$$
\begin{aligned}
n & =\frac{\alpha^{2}(R-V W)}{\alpha^{2} \Gamma\left(R+W^{2}\right)+(V+W)^{2}}(\gamma \tilde{t}-\beta \tilde{f}) \\
t & =\frac{\alpha^{2}\left(R+W^{2}\right)}{\alpha^{2} \Gamma\left(R+W^{2}\right)+(V+W)^{2}}(\gamma \tilde{t}-\beta \tilde{f}) \\
s_{n} & =\frac{\alpha W(V+W)}{\alpha^{2} \Gamma\left(R+W^{2}\right)+(V+W)^{2}}(\gamma \tilde{t}-\beta \tilde{f}) \\
s_{t} & =\frac{\eta(V+W)}{\alpha^{2} \Gamma\left(R+W^{2}\right)+(V+W)^{2}}(-\gamma \tilde{t}+\beta \tilde{f}) \\
s_{f} & =\frac{\kappa(V+W)}{\alpha^{2} \Gamma\left(R+W^{2}\right)+(V+W)^{2}}(\gamma \tilde{t}-\beta \tilde{f}) .
\end{aligned}
$$

\subsubsection{Interpretation}

The various control variables $n, t, f, s_{n}, s_{t}$, and $s_{f}$ depends linearly on the goals $\tilde{t}$ and $\tilde{f}$. The matrices expressing these dependencies are themselves functions of the model parameters $\alpha, \beta$, $\gamma \ldots$ These results need to be interpreted by analyzing the dependencies in $\tilde{t}$ and $\tilde{n}$. In doing so, we will emphasize the different channels available for the unconscious $\mathrm{U}$, in driving other agents in his direction.

In this model, the behavior of the agent is dictated by the complementarity $f+t=1$, and depends crucially on the ratio $R=\frac{\kappa^{2}+\eta^{2}}{\alpha^{2}}$. This ratio measures the relative efficiency of the two channels the unconscious agent can use to affect the conscious' behavior.

Actually, when $R$ is large, the conscious is more sensitive to the "intellectual" direct strains $s_{t}$ and $s_{f}$ and the unconscious will focus on this mean of action. If the ratio is small, the unconscious will preferably act on the conscious through the indirect strain $s_{n}$, i.e. body needs. The necessity to choose between these two channels, and the different results it will lead to, are the direct consequence of the time complementarity between $t$ and $f$, as we shall see.

The activity level $t$ is straightforward to analyze. It depends positively on $\tilde{t}$ and negatively on $\tilde{f}$. When $\tilde{t}$ raises, the unconscious is able to induce a higher activity through two ways. First by reducing the strain $s_{t}$, and second by reducing the physical need via a decrease in the strain $s_{n}$. For the same reason $t$ depends negatively on $\tilde{f}$. The unconscious raises $f$ through $s_{f}$ and $s_{n}$, which reduces $t$ by complementarity.

However, and interestingly, these unambiguous results can be achieved in two different ways, leading to an ambiguous sign for the physical need $n$, as can be seen in the results. As explained above, the unconscious has two different channels of action on the conscious, and the relative efficiency of these channels is measured by $R$.

When $R>V W$, the direct action of the unconscious on the conscious is more efficient than the indirect one, i.e. the manipulation of the body, and $t$ depends positively on $\tilde{t}$.

It will prove more efficient for the unconscious to induce a higher strain $s_{t}$ on the conscious, rather than acting through the physical need. As consequence, a higher $\tilde{t}$ implies a higher $t$, as seen above, as well as a reduced $f$. The indirect channel being not efficient enough, the unconscious is unable to reduce the resulting $n$ through the strain $s_{n}$, and $n$ rises.

On the other hand, when $R<V W$, when $\tilde{t}$ increases, the unconscious will rather use the indirect channel to decrease the need $n$ and induce a higher $t$. 
For complementarity reasons, when $R>V W$, the task $t$ depends negatively on $\tilde{f}$. In this case, in order to increase $n$, the unconscious increases the strain $s_{f}$ that reduces $t$, that in turn increase $f$ and reduces $n$. On the contrary, if $R<V W$, the unconscious will act through the body and increase the need $n$, which reduces $t$ and increases $f$.

This model therefore describes two kinds of agents. The first one (case $R>V W$ ) is driven away from the conscious equilibrium for the task $t$. But doing so, he is not able to to satisfy the physical need $n$ and feels the effect of such an effort. One may conjecture that, in a dynamic perspective, this equilibrium will not be sustainable in the long run. Symmetrically, it could also describe an agent prevented from working or performing some activities. This agent will rather spend his time with the complementary activity such as smoking, eating... This last possibility is sustainable.

The second type of agent (case $R<V W$ ) can also be driven away from the conscious equilibrium in an hyperactivity phase, through a kind of "anesthesia". The rise in his level of activity, for instance, will not be followed by a feeling of disequilibrium. This may be sustainable a certain amount of time, but may lead to a breakdown in the longer run.

\subsection{Case when $f$ and $t$ are independent}

When we relax the time binding condition and allow the control variables $f$ and $t$ to be independent, the utility functions

$$
\begin{aligned}
U_{\mathrm{B}} & =-\frac{1}{2}(n+f)^{2}-\alpha n s_{n} \\
U_{\mathrm{U}} & =-\frac{1}{2}\left(\beta(f-\tilde{f})^{2}+\gamma(t-\tilde{t})^{2}+s_{n}^{2}+s_{f}^{2}+s_{t}^{2}\right) \\
U_{\mathrm{C}} & =-\frac{1}{2}\left(t^{2}+\delta n^{2}\right)-\nu n t-\kappa s_{f}(f-\tilde{f})-\eta s_{t}(t-\tilde{t})
\end{aligned}
$$

lead to the equivalent matricial form

$$
\begin{aligned}
U_{\mathrm{B}}^{\prime} & =-\frac{1}{2}{ }^{t} X_{1} X_{1}-{ }^{t} X_{1} A_{12}^{(1)} X_{2}-{ }^{t} X_{1} A_{13}^{(1)} X_{3}-\frac{1}{2}{ }^{t} X_{3} A_{33}^{(1)} X_{3} \\
U_{\mathrm{U}}^{\prime} & =-\frac{1}{2}{ }^{t} X_{2} A_{22}^{(2)} X_{2}-\frac{1}{2}{ }^{t}\left(X_{3}-\tilde{X}_{3}\right) A_{33}^{(2)}\left(X_{3}-\tilde{X}_{3}\right) \\
U_{\mathrm{C}}^{\prime} & =-\frac{1}{2}{ }^{t} X_{3} A_{33}^{(3)} X_{3}-\frac{1}{2} X_{1} A_{11}^{(3)} X_{1}-{ }^{t} X_{3} A_{31}^{(3)} X_{1}-{ }^{t} X_{2} A_{23}^{(3)}\left(X_{3}-\tilde{X}_{3}\right)
\end{aligned}
$$

where the control variables for the agents are now concatenated in the following vectors:

$$
X_{1}=(n), \quad X_{2}=\left(\begin{array}{c}
s_{n} \\
s_{t} \\
s_{f}
\end{array}\right), \quad X_{3}=\left(\begin{array}{c}
t \\
f
\end{array}\right)
$$

The matrices are now given by 


$$
\begin{array}{ccc}
A_{12}^{(1)}=\left(\begin{array}{lll}
\alpha & 0 & 0
\end{array}\right), & A_{13}^{(1)}=\left(\begin{array}{ll}
0 & 1
\end{array}\right), & A_{33}^{(2)}=\left(\begin{array}{ll}
\beta & 0 \\
0 & \gamma
\end{array}\right) \\
A_{33}^{(3)}=\left(\begin{array}{ll}
1 & 0 \\
0 & 0
\end{array}\right), & A_{11}^{(3)}=(\delta), & A_{31}^{(3)}=\left(\begin{array}{l}
\nu \\
0
\end{array}\right) \\
A_{23}^{(3)}=\left(\begin{array}{ll}
0 & 0 \\
\eta & 0 \\
0 & \kappa
\end{array}\right), & A_{33}^{(1)}=\left(\begin{array}{ll}
0 & 0 \\
0 & 1
\end{array}\right), & A_{22}^{(2)}=\left(\begin{array}{lll}
1 & 0 & 0 \\
0 & 1 & 0 \\
0 & 0 & 1
\end{array}\right) .
\end{array}
$$

The optimization problem is exactly the same as in the previous case, provided we cancel all the constant matrices $E_{i}$ for $i=1,2,3$. Using the same procedure as before, we get

$$
\begin{aligned}
& X_{2}=\left(A_{22}^{(2)}+{ }^{t} K A_{33}^{(2)} K\right)^{-1}{ }^{t} K A_{33}^{(2)} \tilde{X}_{3} \\
& X_{3}=K\left(A_{22}^{(2)}+{ }^{t} K A_{33}^{(2)} K\right)^{-1}{ }^{t} K A_{33}^{(2)} \tilde{X}_{3} \\
& X_{1}=L\left(A_{22}^{(2)}+{ }^{t} K A_{33}^{(2)} K\right)^{-1}{ }^{t} K A_{33}^{(2)} \tilde{X}_{3}
\end{aligned}
$$

with

$$
K=-\left(A_{33}^{(3)}+A_{31}^{(1)} A_{11}^{(3)} A_{13}^{(1)}-A_{31}^{(3)} A_{13}^{(1)}-A_{31}^{(1)} A_{13}^{(3)}\right)^{-1}\left(A_{32}^{(3)}+A_{31}^{(1)} A_{11}^{(3)} A_{12}^{(1)}-A_{31}^{(3)} A_{12}^{(1)}\right)
$$

and

$$
L=-\left(A_{12}^{(1)}+A_{13}^{(1)} K\right)
$$

A more precise description of the equilibrium can be given by the following formulae. Set

$C=\kappa^{2}+\delta \eta^{2}, \quad B=\beta \alpha^{2}+1, \quad N=\nu^{2} \eta^{2}+\gamma \eta^{2} \kappa^{2}+\kappa^{2}, \quad \Delta=\delta-\nu^{2}, \quad E=\eta^{2} \delta^{2}+\nu^{2} \kappa^{2}$, and

$$
D=\left(\Delta^{2}+\gamma E\right) B+\beta N .
$$

Note that, in our conventions, all these quantities are nonnegative. The solutions are explicitly given by

$$
\begin{aligned}
n & =-\left(\frac{\gamma \nu C B}{D}\right) \tilde{t}-\left(\frac{\beta N}{D}\right) \tilde{f} \\
t & =\left(\frac{\gamma\left(E B+\beta \eta^{2} \kappa^{2}\right)}{D}\right) \tilde{t}+\left(\frac{\beta \nu C}{D}\right) \tilde{f} \\
f & =\left(\frac{\gamma \nu C}{D}\right) \tilde{t}+\left(\frac{\beta\left(N+\alpha^{2}\left(\Delta^{2}+\gamma E\right)\right)}{D}\right) \tilde{f} \\
s_{n} & =\left(\frac{\alpha \beta \gamma \nu C}{D}\right) \tilde{t}-\left(\frac{\alpha \beta\left(\Delta^{2}+\gamma E\right)}{D}\right) \tilde{f} \\
s_{t} & =-\left(\frac{\gamma \eta\left(\delta \Delta B+\beta \kappa^{2}\right)}{D}\right) \tilde{t}+\left(\frac{\beta \eta \nu\left(\kappa^{2} \gamma-\Delta\right)}{D}\right) \tilde{f} \\
s_{f} & =\left(\frac{\gamma \kappa \nu\left(\eta^{2} \beta-\Delta B\right)}{D}\right) \tilde{t}-\left(\frac{\beta \kappa\left(\delta \gamma \eta^{2}+\Delta\right)}{D}\right) \tilde{f} .
\end{aligned}
$$




\subsubsection{Interpretation}

Removing the constraint $f+t=1$ allows more freedom for the conscious. Without the trade off between the activity and satisfying the need, $n$ depends negatively on $\tilde{f}$. This is straightforward: the unconscious directly drives the body toward his goals. On the other hand, $n$ depends also negatively on $\tilde{t}$. Actually, the unconscious will lead the conscious to a higher level of activity by inducing a higher feeding $f$, and thus reducing the need $n$.

For similar reasons, $t$, the conscious' task, will depend positively on $\tilde{t}$ and $\tilde{f}$. A higher $\tilde{f}$ will lead to a higher $n$, a lower $n$ and in turn a higher task level $t$.

This departs from the previous model: in the absence trade off between $f$ and $t$, the unconscious is able to manipulate both the activity level and the feeding at the same time. This possibility would for instance describe a situation in which both feeding and working are possible at the same time, writing and smoking, or doing some sport and snacking, etc...

This can have enhancement effects: when $\tilde{f}$ and $\tilde{t}$ are non contradictory and have the same sign, it can induce high worker-high smoker profiles, or depressive effects, such as no task-no feeding, each effect boosting the other.

However, it can also describe neutralization behaviors, when goals are contradictory, such as $\tilde{f}<0$ and $\tilde{t}>0$, for example. Some anorexic pattern could exemplify this type of situations. Under a dynamic description, a high level of activity could be quickly deterred by the impossibility for the agent to satisfy the body's needs.

For the sake of precision, let us detail the mechanisms at stakes here.

whatever the parameters of the model, $n$ depends negatively on $\tilde{f}$. When $\mathrm{U}$ acts, the magnitude of the dependency increases with $\beta$, the weight assigned by $\mathrm{U}$ to $f$. More surprisingly, $n$ reacts negatively to $\tilde{t}$. This is the clue to U's underground activity. Actually, when $\tilde{t}>0$, the unconscious aims at raising the conscious' task $t$, when $\mathrm{C}$ would rather set $t=0$. For this purpose, $\mathrm{U}$ uses an indirect channel through the body's action, when $\alpha \neq 0$. Inducing a negative strain on $\mathrm{B}$ will result in a lower $n$, and a higher $t$, as wanted by $\mathrm{U}$. In other words, the unconscious is able to calm down the body' need to stimulate the conscious' activity. On the contrary, when $\tilde{t}<0$, U can increase the body's need to damper the conscious' task. In a psychological context of neurosis, this a common behavior of hyperactivity or failure: a subject feels a modification of his physical state, that can either boost or impair a conscious project.

The variable $t$ reacts positively to $\tilde{f}$ and $\tilde{t}$. The unconscious manipulates $\mathrm{C}$ to lead his actions toward U's goals $\tilde{t}$. The dependence of $t$ in $\tilde{t}$ is straightforward. U influences $\mathrm{C}$ directly through $s_{t}$. The goal $\tilde{t}$ is not fully reached, since the unconscious suffers also a loss from the strain $s_{t}$. The dependence of $t$ in $\tilde{f}$ is indirect. It results in a crossed interaction between $\mathrm{U}, \mathrm{B}$ and $\mathrm{C}$. As a consequence of the strain $s_{f}$ imposed by $\mathrm{U}$ on $\mathrm{C}$, when $\tilde{f}$ raises, $f$ will increase as a consequence. The increase of $f$ will in turn reduce the signal $n$ sent by the body, and allow the conscious to set a higher level of task $t$ (since the cost of $n$ in $U_{\mathrm{C}}$, that is $-\nu n t$, dampens).

The variable $f$ depends positively on $\tilde{f}$ and $\tilde{t}$. The proof is the same as the one for $t$. It suffices to exchange the roles of $\tilde{f}$ and $\tilde{t}$.

For $\alpha \neq 0$, the control variable $s_{n}$ depends positively on $\tilde{t}$ an negatively on $\tilde{f}$. Indeed, for $\tilde{n}>0$, it is beneficial to $\mathrm{U}$ to send a higher strain to the body, so that the body's need $n$, and thus $f$ the conscious answer to it will increase. If $\tilde{t}>0$, the unconscious agent will reduce the body need $n$, through a negative strain $s_{n}$. It will induce a higher task $t$ for the conscious agent, as wanted by $\mathrm{U}$. Trivially, if one has $\alpha=0$, Agent $\mathrm{U}$ has no influence on $B$ and, as expected, the strain $s_{n}$ is zero. 
The strain $s_{t}$ depends positively on $-\eta \tilde{t}$. We remind that one has $-\eta \tilde{t}>0$ as explained in Section 1. As expected, more $|\tilde{t}|$ raises, the more U sends a strain $s_{t}$ on the conscious agent to lead the latter to raise his action $t$. The dependance of $s_{t}$ on $\tilde{f}$ is ambiguous, that is, its sign depends on the various parameters. For $\kappa=0$, if $\tilde{f}$ raises, Agent $\mathrm{U}$ will induce higher need $n$ for B, through $s_{n}$, so that $f$ raises. However, the increase in $n$ will lead $\mathrm{C}$ to reduce his task $t$. Agent U, will thus be led to reduce $s_{t}$ to stabilize $t$. However if $\kappa$ is set a higher positive level, Agent $\mathrm{U}$ can now directly influence the level of $f$ and thus has the tendency to reduce its use of $s_{t}$.

For complete similar reasons, $s_{f}$ depends positively on $-\kappa \tilde{f}$ and ambiguously on $\tilde{t}$.

The ambiguous signs on the strain translate the fact that $\mathrm{U}$ despite his strategic domination, has to adapt his behavior to the interaction between B and C. In absence of the term $-\nu n t$ in $U_{\mathrm{C}}$, Agent $U$ could set his strains $s_{f}$ and $s_{t}$ to adjust $f$ and $t$ independently one from the other. In the presence of $-\nu n t$, as we saw, the strains act on both $f$ and $t$ in a dependent way. The adjustment has also to be done dependently. As seen above, in order to active U's goals, it can be useful to reduce a strain associated to one of the variable to increase indirectly the other one.

\section{General case: Several interacting structures.}

Let us now present a model for several interacting agents where some may have strategic advantages over others. We also give a general resolution method for this type of model.

The relations of strategic domination between agents are described by an oriented graph. For $f$ agents, let us consider the graph $\Gamma$ whose vertices are the integers from 1 to $f$, each vertex corresponding to an agent. If agent $i$ has a strategic advantage over agent $j$, we draw an oriented edge from $i$ to $j$ and write $i \rightarrow j$. For later purpose if there exists an oriented path from $i$ to $j$, we write the relation $i \longmapsto j$. In this situation, we will state that agent $i$ dominates directly or indirectly agent $j$ or, equivalently, that agent $j$ is subordinated to agent $i$. On the other hand, we will write $j \& i$ if there is no oriented path from $i$ to $j$, where it is always understood that $i \neq j$.

We will merely consider connected graphs without loops. In the model of Section 2, for instance, if $\mathrm{B}$ is number $1, \mathrm{U}$ is number 2 , and $\mathrm{C}$ is number 3 , we have: $3 \rightarrow 1,2 \rightarrow 1$, and $2 \rightarrow 3$. So we also have $3 \longmapsto 1,2 \longmapsto 1$, and $2 \longmapsto 3$. Note that here the relation $2 \longmapsto 1$ is obtained via the edge $2 \rightarrow 1$, but also by the path $2 \rightarrow 3 \rightarrow 1$. This means that the unconscious can manipulate the body directly or indirectly through the conscious agent. We note here that the model presented in [FL] would be encoded in an unbounded graph of type $1 \rightarrow 2 \rightarrow 3 \rightarrow \cdots$ in which agent 1 is the "long-run self" and the other ones are the "short-run selves". Let $X_{i} \in R^{n_{i}}$ be the agent $i$ 's vector of control variables. The vector $\tilde{X}_{j}^{(i)} \in R^{n_{i}}$ is the vector of the goals for the variables $X_{j}$, as expected by agent $i$. We normalized the vector $\tilde{X}_{j}^{(i)}$ to 0 . In other terms, agent $i$ wishes to achieve $X_{i}=0$ and $X_{j}=\tilde{X}_{j}^{(i)}$.

Agent $i^{\prime} s$ utility is given by:

$$
\begin{aligned}
U_{i}= & -\frac{1}{2} X_{i} A_{i i}^{(i)} X_{i}-\frac{1}{2} \sum_{j \longleftarrow i}{ }^{t}\left(X_{j}-\tilde{X}_{j}^{(i)}\right) A_{j j}^{(i)}\left(X_{j}-\tilde{X}_{j}^{(i)}\right) \\
& -\sum_{j \hookleftarrow i}{ }^{t} X_{i} A_{i j}^{(i)} X_{j}-\sum_{j \nprec i}{ }^{t}\left(X_{i}-\tilde{X}_{i}^{(j)}\right) A_{i j}^{(i)} X_{j}-{ }^{t} X_{i} E_{i}
\end{aligned}
$$


The term $-\frac{1}{2}^{t} X_{i} A_{i i}^{(i)} X_{i}$ is the utility of Agent $i$ per se, that is to say agent $i$ 's utility in absence of any interaction. The variables $X_{i}$ are normalized such that the matrix $A_{i i}^{(i)}$ is a $f_{i} \times n_{i}$ diagonal matrix with coefficients equal 1 or 0 .

The term $\sum_{j \leftarrow i}{ }^{t}\left(X_{j}-\tilde{X}_{j}^{(i)}\right) A_{j j}^{(i)}\left(X_{j}-\tilde{X}_{j}^{(i)}\right)$ models agent $i^{\prime} s$ loss of utility if the actions $X_{j}$ of its subordinate agents depart from $\tilde{X}_{j}^{(i)}$. The $f_{j} \times n_{j}$ matrix $A_{j j}^{(i)}$ of parameters is of course symmetric.

The next terms $\sum_{j \leftarrow i}{ }^{t} X_{i} A_{i j}^{(i)} X_{j}-\sum_{j \nprec i}{ }^{t}\left(X_{i}-\tilde{X}_{i}^{(j)}\right) A_{i j}^{(i)} X_{j}$ describe the impact induced on agent $i$ 's utility by agent $j^{\prime} s$ action. $j \longleftarrow i$ can be seen as the resulting collateral damage to agent $i$ induced by agent $j^{\prime} s$ action. In our model, agent $j$ can't "see" the agents to whom he is subordinated. The second term models the strain imposed by agent $j$ to agent $i$ in order to achieve its own objectives for $X_{i}$. Note that it does not matter to write $\sum_{j \nprec i}{ }^{t} X_{i} A_{i j}^{(i)} X_{j}$ instead of $\sum_{j \nprec i}^{t}\left(X_{i}-\tilde{X}_{i}^{(j)}\right) A_{i j}^{(i)} X_{j}$. The linear term in $X_{j}$ disappears during the resolution. By convention, for the $n_{i} \times n_{j}$ parameters matrices $A_{i j}^{(i)}$, we will write ${ }^{t} A_{i j}^{(i)}=A_{j i}^{(i)}$.

The last term ${ }^{t} X_{i} E_{i}$ models the loss or gain of utility caused by possible external perturbations summarized by the vector $E_{i} \in R^{n_{i}}$.

Let us now describe the general process which leads to the resolution of the model. For all $j$ 's such that $j \longleftarrow i$, the variable $X_{j}$ will depend on $X_{i}$ as agent $j$ is dominated at least indirectly by agent $i$. The maximization equation for agent $i$ 's utility yields directly

$$
\begin{aligned}
& \left(A_{i i}^{(i)}+\sum_{j \hookleftarrow i} t^{t}\left(\frac{\partial X_{j}}{\partial X_{i}}\right) A_{j i}^{(i)}\right) X_{i}+\sum_{j \hookleftarrow i}\left(A_{i j}^{(i)}+{ }^{t}\left(\frac{\partial X_{j}}{\partial X_{i}}\right) A_{j j}^{(i)}\right) X_{j}+\sum_{j \nprec i} A_{i j}^{(i)} X_{j} \\
= & \left(E_{i}+\sum_{j \longleftarrow i} t\left(\frac{\partial X_{j}}{\partial X_{i}}\right) A_{j j}^{(i)} \tilde{X}_{j}\right) .
\end{aligned}
$$

To solve the model, the matrices $\frac{\partial X_{j}}{\partial X_{i}}$ must be found first. These matrices are zero when agent $j$ is dominated neither directly nor indirectly by agent $i$. The non zero matrices can be computed recursively way back along the paths provided by the graph from the most dominated agents to the most dominating ones.

We need now to introduce further notations. For two agents $i$ and $j$ such that $i \longmapsto j$, we set $\hat{M}_{j i}=\left(\frac{\partial X_{j}}{\partial X_{i}}\right)$. If $i \rightarrow j$, we define $M_{j i}$ to be the derivative of $X_{j}$ with respect to $X_{i}$ in the case where only the direct domination in considered, i.e. all other paths between $i$ and $j$ have been removed. We also set $M_{i i}=I d_{n_{i}}$. This setting lead to the following recursive formula

$$
\frac{\partial X_{j}}{\partial X_{i}}=\hat{M}_{j i}=\sum_{j \longleftarrow \ell \leftarrow i} \hat{M}_{j \ell} M_{\ell i}
$$

which is merely the chain rule. Moreover, note that if there agent $i$ dominates neither directly nor indirectly agent $j, \hat{M}_{j i}=0$ 
We can now proceed to solve the problem. In the last equation above, we replace the expressions for the derivatives by our recursive formula, and obtain:

$$
\begin{aligned}
& \left(A_{i i}^{(i)}+\sum_{j \longleftarrow i}{ }^{t}\left(A_{i j}^{(i)} \hat{M}_{j i}\right)\right) X_{i}+\sum_{j \longleftarrow i}\left(A_{i j}^{(i)}+{ }^{t} \hat{M}_{j i} A_{j j}^{(i)}\right) X_{j}+\sum_{j \nprec i} A_{i j}^{(i)} X_{j} \\
= & \left(E_{i}+\sum_{j \hookleftarrow i}{ }^{t} \hat{M}_{j i} A_{j j}^{(i)} \tilde{X}_{j}\right)
\end{aligned}
$$

Let $k$ be such that $k \rightarrow i$. We differentiate again the previous equation with respect to $X_{k}$, to obtain:

$$
\begin{aligned}
& \sum_{\ell \leftarrow k}\left(A_{i i}^{(i)}+\sum_{j \leftarrow i}\left(A_{i j}^{(i)} \hat{M}_{j i}\right)\right) \hat{M}_{i \ell} M_{\ell k}+\sum_{j \leftarrow i}\left(\left(A_{i j}^{(i)}+{ }^{t} \hat{M}_{j i} A_{j j}^{(i)}\right) \sum_{\substack{\ell \leftarrow k \\
j \leftarrow \ell \text { or } j=\ell}} \hat{M}_{j l} M_{l k}\right) \\
& +\sum_{\substack{\ell \hookleftarrow k \ell \ell \rightarrow i \\
j \leftarrow i \\
j \longleftarrow \text { or } j=\ell}} A_{i j}^{(i)}\left(\hat{M}_{j l} M_{l k}\right) \\
= & -A_{i k}^{(i)}
\end{aligned}
$$

The last equality leads, for any $i \leftarrow k$, to

$$
\sum_{\ell \leftarrow k}\left(+\sum_{\substack{j \longleftarrow i \\ j \hookleftarrow \ell \text { or } j=\ell}}^{\left(A_{i i}^{(i)}+\sum_{j \longleftarrow i}{ }^{t}\left(A_{i j}^{(i)} \hat{M}_{j i}\right)\right) \hat{M}_{i \ell}}\right.
$$

where $\delta_{\ell i}$ stands for the Kronecker symbol.

We thus obtain a linear system of equations, in which the unknowns are the matrices $M_{\ell k}$ for $\ell \leftarrow k$ and the coefficients are functions of the matrices $\hat{M}_{j \ell}$ and $\hat{M}_{i \ell}$ with $j, i \leftarrow \ell$. The system is given by the matricial equation

$$
\Theta_{k} M_{k}=-A_{k}
$$

where we defined $M_{k}$ and $A_{k}$ as block vectors by setting

$$
M_{k}=\left[\begin{array}{c}
M_{\ell_{1} k} \\
\vdots \\
M_{\ell_{r_{k}} k}
\end{array}\right] \quad \text { and } \quad A_{k}=\left[\begin{array}{c}
A_{\ell_{1} k}^{\left(\ell_{1}\right)} \\
\vdots \\
A_{\ell_{r_{k} k}}^{\left(\ell_{r_{k}}\right)}
\end{array}\right]
$$

Here $\ell_{s}$ with $1 \leq s \leq r_{k}$ are the $r_{k}$ agents directly dominated by Agent $k$. Moreover $\Theta_{k}$ the block matrix which block coefficients are given by

$$
\left(\Theta_{k}\right)_{i \ell}=\left(A_{i i}^{(i)}+\sum_{j \longleftarrow i}{ }^{t}\left(A_{i j}^{(i)} \hat{M}_{j i}\right)\right) \hat{M}_{i \ell}+\sum_{\substack{j \longleftarrow i \\ j \longleftarrow \ell \text { or } j=\ell}}\left(A_{i j}^{(i)}+{ }^{t} \hat{M}_{j i} A_{j j}^{(i)}\right) \hat{M}_{j \ell}+\left(1-\delta_{\ell i}\right) \sum_{\substack{j \nLeftarrow i \\ j \hookleftarrow \ell \text { or } j=\ell}} A_{i j}^{(i)} \hat{M}_{j \ell} .
$$


As the matrix $\Theta_{k}$ only depends on the matrices $M_{j}$ with $k \longmapsto j$, one can recursively solve the equations $\Theta_{k} M_{k}=-A_{k}$ if one assumes that the parameters are such that the matrices $\Theta_{k}$ are invertible.

When $k$ is one of the penultimate edge (in other terms the edges of agents dominating directly whose which are themselves not dominating anyone), for any $i \leftarrow k$ and $\ell \leftarrow k$ one has

$$
\left(\Theta_{k}\right)_{i \ell}=A_{i i}^{(i)} \hat{M}_{i \ell}+\left(1-\delta_{\ell i}\right) A_{i \ell}^{(i)} \hat{M}_{\ell \ell}
$$

Given that we have $\hat{M}_{\ell \ell}=M_{\ell \ell}=I d_{n_{\ell}}$ and $\hat{M}_{i \ell}=0$ for $\ell \neq i$, one gets

$$
\left(\Theta_{k}\right)_{i \ell}=\delta_{\ell i} A_{i i}^{(i)}+\left(1-\delta_{\ell i}\right) A_{i \ell}^{(i)}=A_{i \ell}^{(i)}
$$

which is directly given in terms of the initial data.

It suffice now to let $k$ run backward from penultimate edges of the graph and solve recursively the equations. When all matrices $M_{k}$ are determined, one is ultimately able to compute the solutions $X_{i}$ thought Equation (1) above.

Our resolution method can be used, as an example, to solve the model of section 2.3. We considered the graph with $3 \rightarrow 1,2 \rightarrow 1$, and $2 \rightarrow 3$. Agents $\mathrm{B}, \mathrm{U}$, and $\mathrm{C}$ are respectively denoted by 1,2 , and 3 .

The first equation is given for the graph's penultimate edge $k=3$ and $i=\ell=1$.

$$
\left(\Theta_{3}\right)_{11}=A_{11}^{(1)}=I d_{n_{1}} \quad \Longrightarrow \quad M_{3}=\left[M_{13}\right]=-A_{3}=-\left[A_{13}^{(1)}\right] \quad \Longrightarrow \quad \hat{M}_{13}=M_{13}=-A_{13}^{(1)} .
$$

The second and last step for $k=2$. For $i, \ell \in\{1,3\}$, one computes $\left(\Theta_{2}\right)_{i \ell}$ and gets:

$$
\begin{gathered}
\left(\Theta_{2}\right)_{11}=A_{11}^{(1)} \hat{M}_{11}=A_{11}^{(1)}=I d_{n_{1}} . \\
\left(\Theta_{2}\right)_{13}=A_{11}^{(1)} \hat{M}_{13}+A_{13}^{(1)} \hat{M}_{33}=A_{11}^{(1)} \hat{M}_{13}+A_{13}^{(1)}=-A_{11}^{(1)} A_{13}^{(1)}+A_{13}^{(1)}=0 . \\
\left(\Theta_{2}\right)_{31}=\left(A_{33}^{(3)}+{ }^{t}\left(A_{31}^{(3)} \hat{M}_{13}\right)\right) \hat{M}_{31}+\left(A_{31}^{(3)}+{ }^{t} \hat{M}_{13} A_{11}^{(3)}\right) \hat{M}_{11} \\
=\left(A_{33}^{(3)}+{ }^{t}\left(A_{31}^{(3)} \hat{M}_{13}\right)\right) \times 0+\left(A_{31}^{(3)}+{ }^{t} \hat{M}_{13} A_{11}^{(3)}\right) \hat{M}_{11} \\
=A_{31}^{(3)}+{ }^{t} \hat{M}_{13} A_{11}^{(3)}=A_{31}^{(3)}-A_{31}^{(1)} A_{11}^{(3)} \\
\left(\Theta_{2}\right)_{33}=\left(A_{33}^{(3)}+{ }^{t}\left(A_{31}^{(3)} \hat{M}_{13}\right)\right)+\left(A_{31}^{(3)}+{ }^{t} \hat{M}_{13} A_{11}^{(3)}\right) \hat{M}_{13} . \\
=A_{33}^{(3)}-A_{31}^{(3)} A_{13}^{(1)}-A_{31}^{(1)} A_{13}^{(3)}+A_{31}^{(1)} A_{11}^{(3)} A_{13}^{(1)} .
\end{gathered}
$$

This leads to the matricial equation: $\quad \Theta_{2} M_{2}=-A_{2} \Longleftrightarrow$

$$
\left[\begin{array}{cc}
I d_{n_{1}} & (3) \\
A_{31}^{(3)}-A_{31}^{(1)} A_{11}^{(3)} & A_{33}^{(3)}-A_{31}^{(3)} A_{13}^{(1)}-A_{31}^{(1)} A_{13}^{(3)}+A_{31}^{(1)} A_{11}^{(3)} A_{13}^{(1)}
\end{array}\right] \cdot\left[\begin{array}{l}
M_{12} \\
M_{32}
\end{array}\right]=-\left[\begin{array}{c}
A_{12}^{(1)} \\
A_{32}^{(2)}
\end{array}\right]
$$

which itself leads to the solutions detailed in Section 2. 


\section{Conclusion}

We have developed a general method to solve models of interactions between multiple and possibly strategic agents. Such an approach aims at understanding some of the apparently irrational or incoherent economic agents' behaviors. Describing an economic agent as a system of several rational optimizers may explain why some decisions are biased with respect to a "conscious" point of view. We considered an agent whose "conscious" and "unconscious" goals differ. In such a setting, it appears that the unconscious may influence the conscious either directly, or indirectly via a third agent, the body and its needs. The unconscious may manipulate the body to prevent or enhance the conscious' task, depending whether this task is considered beneficial or undesirable from the unconscious point of view. This three agents model allows the description of behaviors such as craving, excessive smoking, or sleepiness, to delay or dismiss a task. One of our main result stands in the fact that the unconscious' strategic action depends crucially on whether the conscious' actions ("task" and "feeding") are complementary in time. When they are complementary, and if the conscious is not sensitive to the unconscious' messages, the unconscious may drive the conscious towards its goals by blurring physical needs. When they are not complementary, the unconscious may more easily reach his goal by influencing the conscious, be it directly or indirectly. He may make the conscious eat and work according to his goals, and possibly use one variable to enhance the other. For instance, the unconscious may increase the task of the conscious by stressing the physical needs (smoking, use of psychotropic drugs) which will, in certain situation, boost the conscious task. We will consider in a future paper the dynamic version of this model, along with the study of the conditions for an equilibrium and, more interestingly, the possibility of limit cycles leading to cyclic behaviors.

\section{References}

[Ain92] G. Ainslie. Picoeconomics. Cambridge University Press, 1992.

[Ain01] G. Ainslie. Breakdown of Will. Cambridge University Press, 2001.

[Ake91] G. Akerlof. Procrastination and obedience. American Economic Review, 81:1-19, 1991.

[CL03] C. F. Camerer and G. Loewenstein. Behavioral Economics: Past, Present, Future, in Advances in Behavioral Economics. Princeton University Press, 2003.

[FL06] D. Fudenberg and D. K. Levine. A dual self model of impulse control. American Economic Review, 96:1449-1476, 2006.

[GL12] P. Gosselin and A. Lotz. A dynamic model of interactions between conscious and unconscious. Preprint, http://ssrn.com/abstract=2006085, 2012.

[Lot11] A. Lotz. An economic approach to the self: The dual agent. Preprint, http://ssrn.com/abstract=1798999, 2011.

[MT01] S. Mullainathan and R. H. Thaler. Behavioral economics. In International Encyclopedia of Social Sciences, pages 1094-1100. Pergamon Press, 1st edition, 2001.

[Ros05] D. Ross. Economic Theory and Cognitive Science: Microexplanation. Cambridge, MA: MIT Press, 2005.

[Ros09] D. Ross. Integrating the dynamics of multi-scale economic agency. In Harold Kincaid and Don Ross, eds., The Oxford Handbook of Philosophy of Economics. Oxford University Press, 2009. 
[Ros10] D. Ross. Economic models of procrastination. In In C. Andreou \& M. White, eds., The Thief of Time, pages 28-50, 2010.

[Sch78] T. Schelling. Economics, or the art of self-management. American Economic Review, 68:290-294, 1978.

[Sch80] T. Schelling. The intimate contest for self-command. Public Interest, 60:94-118, 1980.

[Sch83] T. Schelling. Self-command in practice, in policy, and in a theory of rational choice. American Economic Review, 74:1-11, 1983.

[Str56] R. Strotz. Myopia and inconsistency in dynamic utility maximization. Review of Economic Studies, 23:165-180, 1956. 